\title{
Modeling of contagious downgrades and its application to multi-downgrade protection
}

\author{
Hidetoshi Nakagawa ${ }^{1}$ \\ ${ }^{1}$ Graduate School of International Corporate Strategy, Hitotsubashi University, 2-1-2 Hitot- \\ subashi, Chiyoda-ku, Tokyo 101-8439, Japan \\ E-mail hnakagawa@ics.hit-u.ac.jp
}

Received March 17, 2010, Accepted April 10, 2010

\begin{abstract}
In this paper, we use a multivariate affine jump process to model the downgrade intensities for several categories of business sector in credit portfolios. Since multivariate affine jump structure enables us to consider self-exciting effects as well as mutually exciting effects, the model can explain the downgrade clusters observed in the Japanese market. Also, we propose a new credit derivative named multi-downgrade protection (MDP) as an application of our model and discuss its fair pricing.
\end{abstract}

Keywords downgrade risk, mutually exciting intensity model, downgrade protection

Research Activity Group Mathematical Finance

\section{Introduction}

In this paper, we present a new modeling of downgrade risk that has been minor to default risk in the literature of credit risk study by using the top-down approach framework introduced by [1]. More specifically, we apply a multivariate affine jump process (see [2]) or a generalized mutually exciting Hawkes model (see [3]) to specify the downgrade intensities for several categories of business sector in credit portfolios.

Fig.1 shows the trajectory of monthly numbers of category-by-category downgrades announced by Rating and Investment Information, Inc. (R\&I) during April 1998 to September 2009. At first glance, we can see that there are more downgrades or downgrade clusters from May 1998 to August 1999, the first half in 2002, and after the second half of 2008 than other months.

One interpretation of these downgrade clusters is that downgrade risk is likely to be contagious in the sense that one downgrade in one category may have some influences not only on the downgrade intensity of the same category but also on those of the other categories. Therefore it looks natural to select a multivariate affine jump process so as to model the downgrade intensities since multivariate affine jump structure enables us to consider self-exciting effects as well as mutually exciting effects. Dynamic rating transition is usually modeled by rating transition intensity matrix, but it seems difficult to use the rating transition matrix to consider dynamic contagion of downgrade risk. Though a non-Markov frameworks is often used for modeling dependence like contagion, some strong assumptions are necessary to achieve the rating transition probability matrix via rating transition intensity matrix as is pointed out in Chap. 6 of [4] and in Chap. 8 of [5].

As an application of our downgrade risk model, we propose a new credit derivative named multi-downgrade

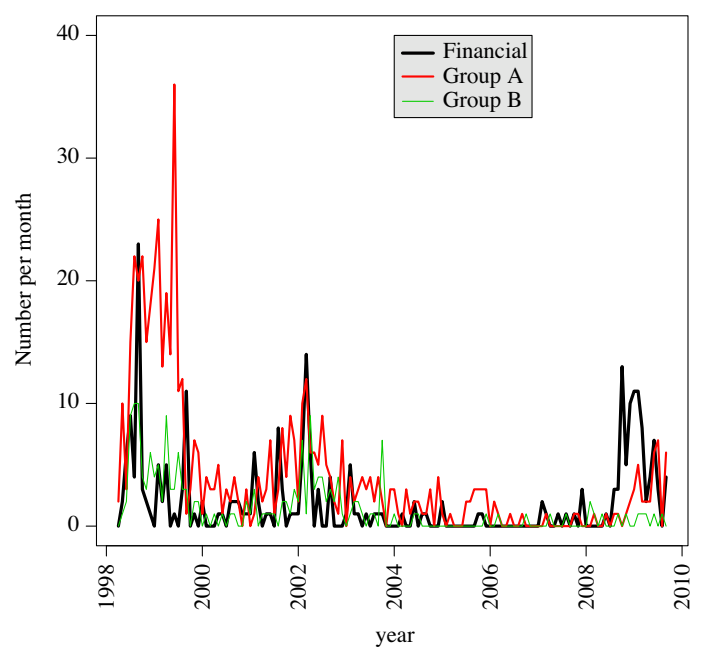

Fig. 1. Trajectory of monthly numbers of category-by-category downgrades announced by R\&I during April 1998 to September 2009. Group A consists of the industry sectors of Communications, Consumer-Cyclical, Industrial and Technology, which seems more influenced by business fluctuation, while Group B consists of Basic Materials, Consumer-Non-cyclical, Energy and Utilities, which seem less influenced by business fluctuation. In all, 1,011 downgrades are observed. There are 263 downgrades are in Fin. category, 562 in Gr.A and 186 in Gr.B.

protection (MDP) that can be a efficient risk hedging tool for large corporate bond portfolios. We also discuss the pricing of MDP under some simple assumptions.

Simply put, MDP is supposed to be the contract that the protection seller pays to the buyer the amount according to the pre-agreed rule over and over again whenever the particular type of downgrade (for example, downgrade from the investment grade to the speculative grade in Gr.A category) happens in the underlying portfolio during the predetermined period. From a practical 
view, MDP seems more useful to manage downgrade risk of the portfolio that consists of a wide variety and number of corporate bonds, because it seems more important in total risk management to consider how many downgrades will happen rather than which bond will be downgraded.

Indeed, we can finally achieve the consequence that the (conditional) expectation of the future downgrade count is essential to evaluate MDP under some assumptions. Also, due to the general theory for multivariate affine jump-diffusion process studied in [6] and [2], we can easily compute the expectation of the future downgrade count based on our downgrade intensities. We show some numerical illustrations of computing the expected future downgrade count that is related to the MDP pricing.

\section{Modeling of downgrade intensities}

We will model contagious downgrade risk with a multivariate affine jump process, which is a slight generalization of self-exciting intensity studied in [2].

Let $(\Omega, \mathcal{F}, P)$ be a complete probability space and $\left(\mathcal{F}_{t}\right)$ be the filtration that makes any processes appeared in this paper adapted.

For some $m \in \mathbb{N}$, let $0\left(=\tau_{0}^{i}\right)<\tau_{1}^{i}<\tau_{2}^{i}<\cdots(i=1$, $\cdots, m)$ be $\left(\mathcal{F}_{t}\right)$-adapted point processes, that is, increasing sequences of $\left(\mathcal{F}_{t}\right)$-stopping times. $\tau_{k}^{i}$ is regarded as the time when $k$-th event of type $i$ happens. Also, $N_{t}^{1}, \cdots, N_{t}^{m}$ are counting processes associated with the point processes $\left\{\tau_{k}^{1}\right\}_{k \in \mathbb{N}}, \cdots,\left\{\tau_{k}^{m}\right\}_{k \in \mathbb{N}}$, respectively.

Suppose that $\left[N^{i}, N^{j}\right]_{t}=0$ almost surely for any $i, j$ $(i \neq j)$.

Next, $L_{t}^{1}, \cdots, L_{t}^{m}$ are $\left(\mathcal{F}_{t}\right)$-adapted pure jump processes whose jump times coincide with those of $N_{t}^{1}$, $\cdots, N_{t}^{m}$. More specifically, for each $i, L_{t}^{i}$ can be characterized by independently and identically distributed random variables $\eta_{1}^{i}, \eta_{2}^{i}, \cdots$, that is,

$$
L_{t}^{i}:=\sum_{k=1}^{N_{t}^{i}} \eta_{k}^{i}
$$

Here we suppose that for any $k \in \mathbb{N}$ and $i=1, \cdots, m$, $\eta_{k}^{i}$ is $\mathcal{F}_{\tau_{k}^{i}}$-measurable.

Then we need to specify the intensity process $X_{t}^{i}$ associated with $N_{t}^{i}$ or equivalently $L_{t}^{i}$, namely, an $\left(\mathcal{F}_{t}\right)$ progressively measurable non-negative process so that the process $M_{t}^{i}$ defined by

$$
M_{t}^{i}:=N_{t}^{i}-\int_{0}^{t} X_{s}^{i} d s
$$

is an $\left(\mathcal{F}_{t}\right)$-martingale.

In this study, we aim to model the intensities so that for any $i, X_{t}^{i}$ can be influenced not only by occurrences of type $i$ event itself (namely "self-exciting" effect), but also by the events other than type $i$ (namely "mutually exciting" effect).

Now, we specify the mutually exciting downgrade intensity model as follows. Let $m=3$. Hereafter, we regard the super-indices 1, 2 and 3 as the downgrade of Fin., Gr.A and Gr.B categories, respectively. We view $L_{t}^{i}$ as the process of the cumulative number of type $i$ events up to time $t$. This means that the jump size $\eta_{k}^{i}$ is equal to the number of type $i$ events which coincidentally happen at time $\tau_{k}^{i}$.

We also assume that $\vec{X}_{t}={ }^{t}\left(X_{t}^{1}, X_{t}^{2}, X_{t}^{3}\right)$ satisfies the following affine-jump type equation.

$$
\left(\begin{array}{c}
d X_{t}^{1} \\
d X_{t}^{2} \\
d X_{t}^{3}
\end{array}\right)=\left(\begin{array}{c}
\kappa^{1}\left(c^{1}-X_{t}^{1}\right) \\
\kappa^{2}\left(c^{2}-X_{t}^{2}\right) \\
\kappa^{3}\left(c^{3}-X_{t}^{3}\right)
\end{array}\right) d t+\sum_{i=1}^{3}\left(\begin{array}{c}
\xi^{1, i} \\
\xi^{2, i} \\
\xi^{3, i}
\end{array}\right) d L_{t}^{i},
$$

where $\kappa^{j}, c^{j},\left\{\xi^{j, i}\right\}_{i=1,2,3}(j=1,2,3)$ and the initial value $X_{0}^{j}$ are all non-negative constant parameters. This specification can be regarded as a little generalization of mutually exciting Hawkes model [3].

Note that $X_{t}^{j}(j=1,2,3)$ can be represented as

$$
X_{t}^{j}=c^{j}+e^{-\kappa^{j} t}\left(X_{0}^{j}-c^{j}\right)+\int_{0}^{t} e^{-\kappa^{j}(t-s)} \sum_{i=1}^{3} \xi^{j, i} d L_{s}^{i} .
$$

Moreover we remark that $X_{t}^{j} \geq \min \left\{c^{j}, X_{0}^{j}\right\} \geq 0$ for any $t \geq 0$ provided that only positive jumps are allowed for every $L_{t}^{i}$, that is, $P\left(\eta_{k}^{i} \geq 0\right)=1$ for any $k \in \mathbb{N}$. This immediately follows from (2) and the assumption that all the parameters are non-negative.

\section{An application: valuation of multi- downgrade protection}

Here we simply define a new derivative named multidowngrade protection (MDP) by the over-the-counter contract that the protection seller has to pay the buyer some amount according to the predetermined rule every time a particular type of downgrade occurs in the underlying portfolio, independent of the individual name downgraded. While [7] and [8] discuss a single downgrade protection, no multi-downgrade case has been considered.

We give the mathematical description of MDP hereafter.

Suppose that $Q$ is a risk-neutral probability measure and fixed. Denote by $r_{t}$ the instantaneous default-free interest rate and by $\Lambda(t, s)=\exp \left(-\int_{t}^{s} r_{u} d u\right)$ the discount factor from time $s(\geq t)$ to time $t$. Then the price denoted by $Z(t, T)$ at time $t$ of the default-free zero-coupon bond with maturity $T$ is specified by $E^{Q}\left[\Lambda(t, T) \mid \mathcal{F}_{t}\right]$.

Let $N_{t}$ and $L_{t}$ be respectively the counting process of the times and the cumulative number of a particular type of downgrade up to time $t$.

Denote by $C_{t}^{T}$ a $\left(\mathcal{F}_{t}\right)$-predictable continuous process that stands for the protection payoff at the time $t$ when one target downgrade happens before the contract maturity $T$.

Then the risk-neutral value $V_{t}^{\cdot, T}$ at time $t$ of a protection leg of MDP with expiration $T(\geq t)$ and payoff process $C_{t}^{T}$ is specified by the following expression.

$$
V_{t}^{\cdot, T}=E^{Q}\left[\int_{t}^{T} \Lambda(t, s) C_{s}^{T} d L_{s} \mid \mathcal{F}_{t}\right] .
$$


Using the integration-by-parts formula, we have

$$
\begin{aligned}
V_{t}^{\cdot, T}=E^{Q} & {\left[\Lambda(t, T) C_{T}^{T} L_{T}^{\cdot} \mid \mathcal{F}_{t}\right]-C_{t}^{T} L_{t} } \\
& +E^{Q}\left[\int_{t}^{T} L_{s} \Lambda(t, s)\left(r_{s} C_{s}^{T} d s-d C_{s}^{T}\right) \mid \mathcal{F}_{t}\right] .
\end{aligned}
$$

For further calculation, we assume the followings.

\section{Assumption 1}

(i) The default-free interest rate $\left\{r_{t}\right\}$ and the particular downgrade count $\left\{L_{t}\right\}$ are independent under $Q$.

(ii) Specify the protection payoff $C_{t}^{T}$ by $Z(t, T) \varphi(t, T)$, where $\varphi(t, T)$ is an $\left(\mathcal{F}_{t}\right)$-adapted process defined by

$$
\varphi(t, T):=\int_{t}^{T} E^{Q}\left[\bar{h}_{u} \mid \mathcal{F}_{t}\right] d u
$$

and the process $\bar{h}_{t}$ follows under $Q$

$$
d \bar{h}_{u}=\alpha\left(\beta-\bar{h}_{t}\right) d t+\sigma_{h} d W_{t}^{h}, \quad \bar{h}_{0}>0,
$$

where $\alpha, \beta$ and $\sigma_{h}$ are positive constants and $W_{t}^{h}$ is $a\left(Q,\left(\mathcal{F}_{t}\right)\right)$-standard Brownian motion that is independent of $r_{t}, L_{t}$ and $Z(t, T)$.

Just remark that we can show that $C_{t}^{T}=Z(t, T) \varphi(t$, $T$ ) is viewed as an approximate difference of the price of corporate zero-coupon bond between before and after downgrade.

It is easy to see that for $s \geq t$

$$
E^{Q}\left[\bar{h}_{s} \mid \mathcal{F}_{t}\right]=\left(\bar{h}_{t}-\beta\right) e^{-\alpha(s-t)}+\beta .
$$

Hence

$$
\varphi(t, T)=\frac{\bar{h}_{t}-\beta}{\alpha}\left(1-e^{-\alpha(T-t)}\right)+\beta(T-t) .
$$

At last, we achieve

$$
\begin{aligned}
d C_{t}^{T} & =\varphi(t, T) d Z(t, T)+Z(t, T) d \varphi(t, T) \\
& =\left(r_{t} C_{t}^{T}-Z(t, T) \bar{h}_{t}\right) d t+\text { (martingale term). }
\end{aligned}
$$

Thanks to Assumption 1 and the trivial consequence that $C_{T}^{T} \equiv 0$ and $\mu^{C}(s, T)=r_{s} C_{s}^{T}-Z(s, T) \bar{h}_{s}$, we can eventually achieve

$$
V_{t}^{\cdot, T}=-C_{t}^{T} L_{t}+Z(t, T) \int_{t}^{T} E^{Q}\left[L_{s} \mid \mathcal{F}_{t}\right] E^{Q}\left[\bar{h}_{s} \mid \mathcal{F}_{t}\right] d s .
$$

The conditional expectation $E^{Q}\left[\bar{h}_{s} \mid \mathcal{F}_{t}\right]$ is given by (3), so the remaining issue to solve is how to compute $E^{Q}\left[L_{s} \mid \mathcal{F}_{t}\right]$.

\section{Numerical example}

In this section, we focus on numerical computation of the expected downgrade count $E\left[L_{t}^{2}\right]$ of Gr.A category under the physical measure $P$. Although we must use the pricing measure $Q$ for MDP valuation, we dare to calculate the expected downgrade count under original probability $P$ because the parameters seen in Table 1 are actually estimated from the historical downgrade records in the Japanese market. Refer to [9] for parameter estimation based on the historical data.

In addition, set $\bar{\eta}^{1}=1.98, \bar{\eta}^{2}=1.6, \bar{\eta}^{3}=1.27$.
Table 1. The maximum likelihood estimates of the parameters of the downgrade intensities (1).

\begin{tabular}{|c|c|c|c|c|c|}
\hline$X_{0}^{1}$ & $\kappa^{1}$ & $c^{1}$ & $\xi^{1,1}$ & $\xi^{1,2}$ & $\xi^{1,3}$ \\
\hline 19.11 & 4.08 & 3.18 & 1.51 & 0.00 & 0.00 \\
\hline \hline$X_{0}^{2}$ & $\kappa^{2}$ & $c^{2}$ & $\xi^{2,1}$ & $\xi^{2,2}$ & $\xi^{2,3}$ \\
\hline 42.09 & 3.26 & 3.17 & 1.17 & 1.00 & 0.82 \\
\hline \hline$X_{0}^{3}$ & $\kappa^{3}$ & $c^{3}$ & $\xi^{3,1}$ & $\xi^{3,2}$ & $\xi^{3,3}$ \\
\hline 24.47 & 4.34 & 1.01 & 0.38 & 0.44 & 1.22 \\
\hline
\end{tabular}

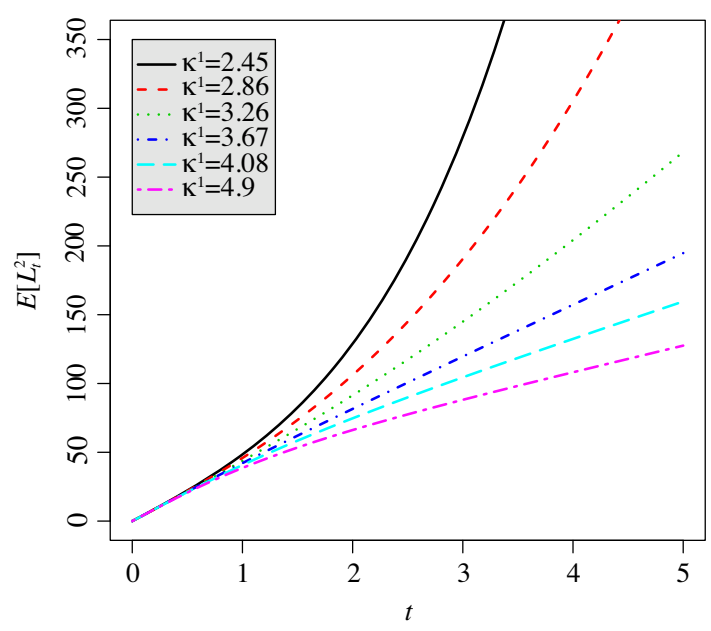

Fig. 2. $E\left[L_{t}^{2}\right]$ for different values of $\kappa^{1}$.

A version of Corollary A.3. of [2] implies that

$$
E\left[L_{t}^{2}\right]=A(0, t)+B(0, t) \cdot{ }^{t}\left(X_{0}^{1}, X_{0}^{2}, X_{0}^{3}, 0,0,0\right),
$$

where $B(0, t)$ and $A(0, t)$ are obtained as below.

The deterministic function $B(0, t)$ is specified by the product of the exponential mapping of the $6 \times 6$-matrix $H$ :

$$
\left(\begin{array}{cccccc}
\bar{\eta}^{1} \xi^{1,1}-\kappa^{1} & \bar{\eta}^{1} \xi^{2,1} & \bar{\eta}^{1} \xi^{3,1} & 1 & 0 & 0 \\
\bar{\eta}^{2} \xi^{1,2} & \bar{\eta}^{2} \xi^{2,2}-\kappa^{2} & \bar{\eta}^{2} \xi^{3,2} & 0 & 1 & 0 \\
\bar{\eta}^{3} \xi^{1,3} & \bar{\eta}^{3} \xi^{2,3} & \bar{\eta}^{3} \xi^{3,3}-\kappa^{3} & 0 & 0 & 1 \\
\text { (The components in the last three rows } \\
\text { are all zero.) }
\end{array}\right.
$$

and $\vec{e}_{5}:={ }^{t}(0,0,0,0,1,0)$.

This exponential mapping $\exp (t H)$ can be numerically calculated by using Runge-Kutta method. On the other hand, we can represent $A(0, t)$ as follows.

$$
A(0, t)=\int_{0}^{t}\left(\kappa^{1} c^{1}, \kappa^{2} c^{2}, \kappa^{3} c^{3}, 0,0,0\right) \cdot\left(\exp (u H) \vec{e}_{5}\right) d u .
$$

We indeed compute $A(0, t)$ by a simple numerical integration to obtain $E\left[L_{t}^{2}\right]$ via (4).

At last, we display some numerical results of comparative analysis for some model parameters that are the long mean reverting speeds $\kappa^{1}$ and $\kappa^{2}$ as well as the mutually exciting components $\xi^{2,1}$ and $\xi^{1,2}$.

First, we change the value of the recursion speed $\kappa^{1}$ of downgrade intensity for Fin. category among the estimate 4.08 times $0.6,0.7,0.8,0.9,1,1.2$ to show the curves of $E\left[L_{t}^{2}\right]$ for $t \in[0,5]$ (see Fig. 2). Since some mutually exciting effect from Fin. to Gr.A is recognized due to the positive estimate $\xi^{2,1}=1.17$, we expect that the value of $\kappa^{1}$ affects time evolution of $E\left[L_{t}^{2}\right]$. We can observe that 
the smaller the value of $\kappa^{1}$ is, the larger the expectation of the target downgrade counts. Small $\kappa^{1}$ means that the downgrade intensity of Fin. remains relatively high even though time passes, so we consider that defaults are likely to happen in Fin. and they are contagious for Gr.A because of the positive mutually exciting effect $\xi^{2,1}$.

Also, the curve shape of $E\left[L_{t}^{2}\right]$ are turned from concave to convex as $\kappa^{1}$ is decreasing. This seems to imply that the level of $\kappa^{1}$ determines whether the downgrade intensity is asymptotically stationary or not.

Second, we change the value of the recursion speed $\kappa^{2}$ of Gr.A to the long-term downgrade intensity among the estimate 3.26 times $0.6,0.7,0.8,0.9,1,1.2$ to show the curves of $E\left[L_{t}^{2}\right]$ (see Fig. 3). As is the same as the $\kappa^{1}$, we can see that the smaller the value of $\kappa^{2}$ is, the larger $E\left[L_{t}^{2}\right]$ and that the curve shape of $E\left[L_{t}^{2}\right]$ are turned from concave to convex as $\kappa^{2}$ is decreasing.

Third, we change the value of the mutually exciting component $\xi^{2,1}$ from 0 to 6 by 1.2 (see Fig. 4). As is expected, we see that the larger $\xi^{2,1}$ becomes, the larger $E\left[L_{t}^{2}\right]$ is.

At last, we change the value of the inverse mutually exciting component $\xi^{1,2}$ (the estimate is zero) from 0 to 1 by 0.2 (see Fig. 5). As is also expected, the larger $\xi^{1,2}$ becomes, the more sharply $E\left[L_{t}^{2}\right]$ increases. A little surprisingly, we see the curve shape of $E\left[L_{t}^{2}\right]$ becomes convex for relatively small value of $\xi^{1,2}$. Anyway larger $\xi^{1,2}$ means that each downgrade in Gr.A causes a larger jump of the downgrade intensity of Fin. category, so downgrades are more likely to occur in Fin. and after all they are contagious for Gr.A due to the positive mutually exciting effect $\xi^{2,1}$.

On the whole, the expected downgrade count is likely to be quite sensitive to the model parameters. This implies the importance of parameter estimation for valuation of MDP.

\section{Acknowledgments}

This research was supported by Grant-in-Aid for Scientific Research (A) No. 20241038 from Japan Society for the Promotion of Science (JSPS). The author also thanks the anonymous reviewer for useful comments.

\section{References}

[1] K. Giesecke and L. R. Goldberg, A top-down approach to multi-name credit, Working paper, Stanford Univ., 2005.

[2] E. Errais, K. Giesecke and L. R. Goldberg, Pricing credit from the top down with affine point processes, Working paper, Stanford Univ., 2006.

[3] A. G. Hawkes, Spectra of some self-exciting and mutually exciting point processes, Biometrika, 58 (1971), 83-90.

[4] D. Lando, Credit Risk Modeling: Theory and Applications, Princeton Univ., 2004.

[5] P. J. Schönbucher, Credit Derivatives Pricing Models: Models, Pricing and Implementation, Wiley, UK, 2003.

[6] D. Duffie, J. Pan and K. Singleton, Transform analysis and asset pricing for affine jump-diffusions, Econometrica, 68 (2000), 1343-1376.

[7] K. Aonuma, An evaluation model for downgrade protection, Jpn J. Indust. Appl. Math., 18 (2001), 627-646.

[8] T. Shimizu, Downgrade protection valuation model (in Japanese), Master thesis of Dep. of Industrial and Management Systems Engineering, Grad. School of Creative Science

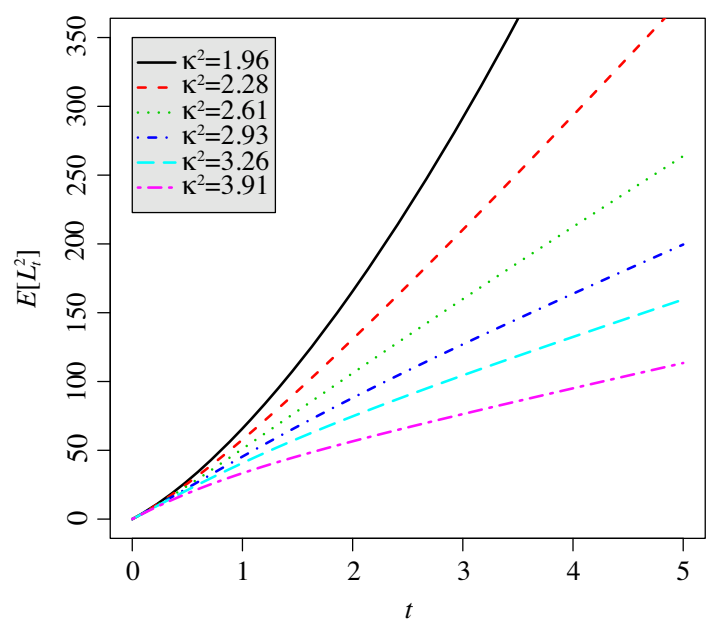

Fig. 3. $E\left[L_{t}^{2}\right]$ for different values of $\kappa^{2}$.

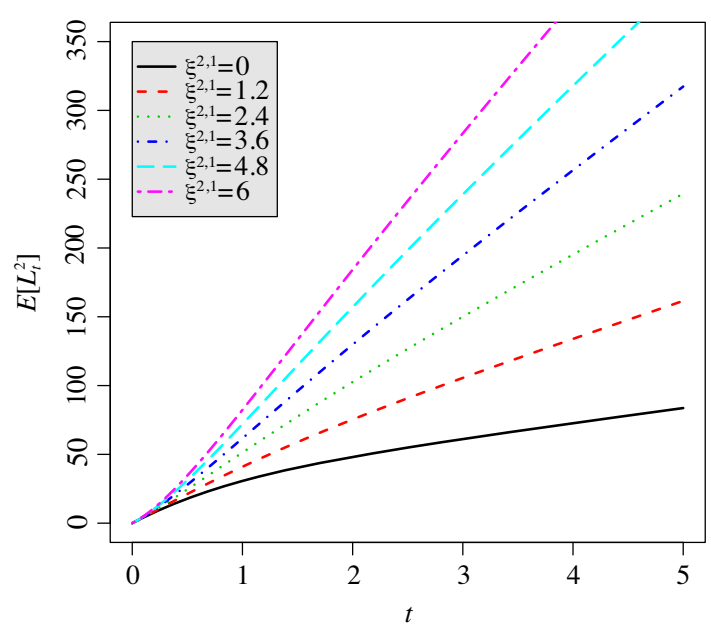

Fig. 4. $E\left[L_{t}^{2}\right]$ for different values of $\xi^{2,1}$.

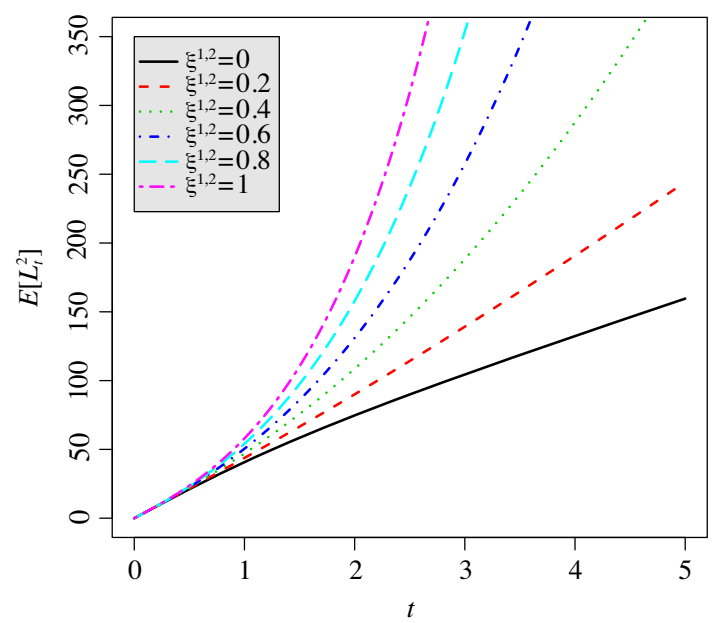

Fig. 5. $E\left[L_{t}^{2}\right]$ for different values of $\xi^{1,2}$.

and Engineering, Waseda Univ., 2003.

[9] H. Nakagawa, Analyses of records of credit rating transition with mutually exciting rating-change intensity model (in Japanese), submitted. 